\title{
A preliminary approach to assess peach fruit texture by Time-Resolved
} Spectroscopy (TRS)

Giovanna Attanasio ${ }^{\text {a }}$, Lorenzo Spinelli ${ }^{\mathrm{b}}$, Alessandro Torricelli ${ }^{\mathrm{c}}$, M. Claudia Piagnani $^{\text {a }}$, Remo Chiozzotto ${ }^{a}$, Daniele Bassi ${ }^{a}$

${ }^{a}$ Dipartimento di Scienze Agrarie e Ambientali, Produzione, Territorio, Agroenergia, Università degli Studi di Milano, via Celoria 2, 20133, Milan, Italy

${ }^{\mathrm{b}}$ Istituto di Fotonica e Nanotecnologie, Consiglio Nazionale delle Ricerche, piazza Leonardo da Vinci 32, I-20133, Milan, Italy

${ }^{\mathrm{c}}$ Politecnico di Milano - Dipartimento di Fisica, piazza Leonardo da Vinci 32, I-20133, Milan, Italy

Key words: Prunus persica, non-destructive test, absorption coefficient, scattering coefficient, food quality

\begin{abstract}
Most fruits can be modeled for their internal composition as a diffusive medium at visible and near-infrared wavelengths. The low absorption in this spectral range allows exploiting VIS/NIR spectroscopic techniques to probe nondestructively the internal food properties. Time-resolved Reflectance Spectroscopy (TRS), in particular, allows the separate but simultaneous estimation of absorption and scattering coefficients. Absorption and scattering properties give very different information about the medium investigated. The pigments and flesh constituents produce characteristic spectral features, so that absorption spectra determination gives information concerning the chemical composition. Light scattering inside the medium is due to local variation of the refractive index, and thus scattering spectrum provides information concerning the structural characteristics of the fruits. The possibility of applying TRS to assess the texture type of peach fruit was tested. To this purpose, an instrument for TRS developed at Politecnico di MilanoDepartment of Physics, was exploited. At least two cultivars for each peach flesh phenotypes (melting, non melting, stony hard and slow melting) and a total of 30 fruits for each cultivar were analyzed over the spectral range $540-940 \mathrm{~nm}$.

The absorption spectra exhibit high values around $550 \mathrm{~nm}$, due to the anthocyanins' absorption features. Furthermore, an absorption peak is visible at $670 \mathrm{~nm}$, linked to the chlorophyll-a content and, then, gives an idea about the fruit ripeness (a higher chlorophyll content corresponds to a less ripe fruit). With the exception of the cultivar 'Iride', particularly rich in anthocyanins, and 'Ghiaccio', a cultivar totally depigmented, the absorption spectra of all the samples are similar. Concerning the scattering properties of peaches, by considering the equivalent density and the scatter power Mie parameters, it is possible to discriminate between three out of four texture types (melting, slow melting and stony hard). Further improvements may lead to a full discrimination in the future.
\end{abstract}

\section{INTRODUCTION}

Until a few years ago, information on internal structure and properties of a fruit could be obtained only by destructive techniques, which are slow and prone to operational errors. Also, these techniques could obviously be applied only to a limited number of 
samples, and the resulting information was extended to the whole batch of fruits, with inaccuracies due to the high levels of biological variability.

The need to assess the quality of each single fruit, in order to improve consumer satisfaction and thus more profitability along the commercial chain, has pushed researchers to develop better non-invasive techniques (Lu and Peng, 2006).

Different techniques have been developed in connection with the quality parameter to be evaluated(Cen et al., 2012; Dahm and Dahm, 2001).

Both the absorption coefficient $\left(\mu_{a}\right)$ and the reduction of the scattering coefficient $\left(\mu_{s}{ }^{\prime}\right)$ may characterize adequately the optical properties of fruits and vegetables (Cen et al., 2012), but absorption and scattering properties of the fruit give very different information about the medium. Absorption is determined by the pigments and flesh constituents that produce characteristic spectral features in the visible and near infrared region, giving information on the chemical composition of the fruit. Scattering is due to local variation of the refractive index inside the medium. Microscopic changes in refractive index caused by membranes, air vacuoles or organelles deviate the photon paths and are ultimately responsible for light diffusion (Cubeddu et al., 2002). Scattering thus provides information concerning the structural characteristics of the fruits. In order to discriminate between the different flesh phenotypes of peaches, or more generally of fruit, it is essential to know the structural characteristics of the sample, and the scattering measurements could have a key role. For this reason scattering information has necessarily to be separated from the absorption information, which is possible with Timeresolved diffuse Reflectance Spectroscopy (TRS).

When a light beam (or streams of photons) impinges on a diffusive medium (the fruit), a small fraction is reflected from the surface (specular reflectance) while the majority of the light penetrates into the fruit. The penetrated light scatters and propagates in different directions in the fruit owing to the local changes in the index of refraction at the interfaces of different cellular structures. Some of the penetrated light is absorbed, some goes through the whole fruit and emerges from the opposite side (transmission), and some scatters back and re-emerges from a region close to the beam incident point: this is often called diffusive reflectance. In a diffusive medium such as a fruit, in the visible and near infrared spectral region, light scattering is stronger than light absorption, so that light can be scattered many times before being either absorbed or re-emitted from the medium: multiple scattering of light (Cubeddu et al., 2002). During this process photons are also absorbed, so that the scattering profile at the surface of the fruit is influenced by, or related to, both absorption and scattering properties of the fruit (Lu and Peng, 2006). The scattering probability per unit length for non-isotropic propagation of photons is described by the reduced scattering coefficient $\mu_{\mathrm{s}}{ }^{\prime}=(1-\mathrm{g}) \mu_{\mathrm{s}}$, where $\mathrm{g}$ is the anisotropy factor, that is the mean cosine of scattering angle and $\mu_{\mathrm{s}}=1 / 1_{\mathrm{s}}, 1_{\mathrm{s}}$ being the photon mean free path between successive scattering events. A direct measure of the photon path length allows to know the effect of scattering, and, since the photon path length is directly related to the photon time-of-flight in the medium, the natural choice is to perform time-resolved measurements (Cubeddu et al., 2002).

TRS consists in determining the delay, the attenuation and the time broadening suffered by a short near infrared laser impulse propagating in a diffusive medium such as fruit flesh. The delay of the collected impulse depends on the light propagation speed inside the medium between source and detector, and the time broadening is a consequence of the different paths of the light inside the medium because of its diffusion (multiple scattering). Finally, the attenuation depends on absorption, which causes loss of photons, 
and on diffusion, which scatters photons along directions different from the direction under consideration (Torricelli et al., 2008).

Compared to conventional optical methods, TRS presents several advantages. First, it allows measurements of the scattering properties of the pulp independent of those of the absorption spectrum. Second, TRS is insensitive to skin color, being most sensitive to internal features. Moreover TRS measurements probe a depth of at least $2 \mathrm{~cm}$ within the flesh (Cubeddu et al., 2002).

\section{MATERIALS AND METHODS}

\section{Peach samples}

The experiment was carried out during the 2011 harvest season. Peaches belonging to four flesh phenotypes (melting, slow melting, non melting and stony hard) were picked in July 2011 from the Experimental orchard of the "Agency for Technology Experimantation and Agroenviromental Research" (ASTRA), Azienda Agricola "Zabina" of Castel San Pietro (Bologna), and from the Experimental Orchard of the University of Milan, Azienda Agricola "F. Dotti" at Arcagna (Lodi). Peach samples were visually inspected and only those free of visual defects were selected for the experiment. Table 1 reports the list of the analyzed accessions.

\section{Ripening parameters}

Immediately after harvest, a total 30 fruits from each accession were preliminarily classified into three maturity stages (less mature, medium mature, more mature), with the index of absorbance difference $\left(\mathrm{I}_{\mathrm{AD}}\right)$ performed by the DA-Meter (Sintéleia Srl, Bologna, Italy). A total of 330 fruits were analyzed. Each fruit was individually numbered.

\section{TRS system}

A device for TRS measurements of food quality, developed at the Politecnico di Milano, Department of Physics, was used. (figure 1). The light source is a supercontinuum fiber laser (SC450-6W, Fianium, UK) providing white-light pico-second pulses, adjustable in power by a variable neutral-density attenuator. A filter wheel loaded with 14 band-pass interference filters is used for spectral selection in the range 540-940 $\mathrm{nm}$. Light is delivered to the sample by means of a multimode graded-index fiber. Diffuse remitted light is collected by $1 \mathrm{~mm}$ fiber. The light is then detected with a photomultiplier (HPM-100-50, Becker \& Hickl, Germany) and the photon distribution of time-of-flight is measured by a time-correlated single-photon counting board (SPC-130, Becker\&Hickl, Germany).

\section{Data analysis}

The temporal profile of the TRS curve was analyzed using a model for the light propagation in diffusive media based on the solution of the Diffusion Equation (Martelli et al., 2009): $R(\rho, t)=A t^{-5 / 2} S\left(\mu_{s}^{\prime} ; t\right) e^{-\mu_{a} v t}$

where $R(\rho, t)$ is the number of photons per unit time $(t)$ and area remitted from the tissue at a distance $\rho$ from the injection point, $\rho$ is the source-detector distance (or inter-fiber distance) and $v$ is the speed of light in the medium. This allows for the simultaneous estimate of $\mu_{a}$ and $\mu_{s}^{\prime}$.

After having retrieved the absorption and scattering coefficients at different wavelengths, composition of fruit constituents and structural parameters can be obtained. In particular, 
absorption spectra can be interpreted as a function of constituent contents by the Beer's law:

$$
\mu_{a}(\lambda)=\sum_{i} C_{i} \varepsilon_{i}(\lambda)=C_{\mathrm{H}_{2} \mathrm{O}} \varepsilon_{\mathrm{H}_{2} \mathrm{O}}+C_{C H L-a} \varepsilon_{C H L-a}
$$

On the other hand, structural parameters can be obtained from the scattering spectra following Mie theory (Mourant et al., 1997; Nilsson et al., 1998):

$$
\mu_{s}^{\prime}(\lambda) \approx a \lambda^{-b}
$$

Where $a$ and $b$ are two parameters linked to the structural properties of the fruit: in particular, $a$ is proportional to the density of the particles causing scattering and $b$ depends on their size.

\section{Statistical data analysis}

The data were tested for differences between flesh phenotypes using the one-way analysis of variance (ANOVA, general linear model) using IBM SPSS statistic software. The differences between flesh phenotypes were tested with Tukey's high significance difference (HSD) test at the 0.05 significance level.

\section{RESULTS AND DISCUSSION}

\section{Absorption}

The absorption spectra were constructed by plotting the value of $\mu_{a}$ as a function of wavelength. Analysing the graphs obtained, it is evident that the absorption spectrum of the peaches between the wavelengths 540 and $940 \mathrm{~nm}$ is dominated, with few exceptions, by the anthocyanin peak around 540-580 nm (Fig. 2). In particular, cultivar 'Iride' scored the highest values in this range (Fig. 2): this is possibly due to the large amount of flesh anthocyanins .

The water peak, around $970 \mathrm{~nm}$, is not visible in the measurements being outside the sensitivity range of the photomultiplier used; however, it is possible to see an increasing of absorption around $940 \mathrm{~nm}$ in every cultivar.

A significant absorption peak at $675 \mathrm{~nm}$, corresponding to chlorophyll-a, is found in all cultivars, with the exception of 'Ghiaccio' (literally: 'ice'), which is characterized by a complete absence of anthocyanins.

\section{Scattering}

A summary of the values of the reduced scattering coefficients based on the average of the 30 fruits tested for every cultivar is showed in figure 3. In general, a greater slope in the reduced scattering profile (like in 'Big Top') shows the presence of smaller particles (either flesh cells or macro-molecules, influencing parameter $b$ ). 'Glohaven' shows a very high dispersion of the value of scattering, from 4 to $20 \mathrm{~cm}^{-1}$. This is due to the fact that some peaches were softer than others: soft fruits tend to show a broader scattering profile than firmer ones (Peng and Lu, 2004).

Figure 4 shows parameter $a$ (on the x-axis), proportional to the density of scattering particles, and parameter $b$ (on the y-axis), proportional to the size of the same particles. Analysis of this graph reveals that the scattering technique has limited capabilities to discriminate between the peach flesh phenotypes. As a matter of fact, melting, slow melting and stony hard peaches are indeed clustered in quite distinct groups, but the other non-melting peaches overlap. 
The TRS device, therefore, although very interesting because it can separate three flesh phenotypes out of four, cannot be considered fully discriminant for distinguishing the diverse flesh phenotypes in peach fruit.

Indeed, by analyzing the two parameters separately, it can be seen that, when the "flesh type" is used as the discriminating parameter for statistical analysis, the "a" parameter discriminates three flesh phenotypes out of four (tab 2), but it does not discriminate melting $\left(16.27 \mathrm{~cm}^{-1}\right)$ from non-melting $\left(16.35 \mathrm{~cm}^{-1}\right)$ phenotypes. If the cultivar is used as discriminating parameter, cultivars with the same flesh type did not fall in the same subset, apart for the stony hard (tab. 3). For "b" parameter, with flesh type as discriminating parameter, it is possible to separate again three phenotypes out of four (tab. 4), but not slow melting (0.36) from non-melting (0.37); again if the cultivar is the discriminating parameter, cultivars of the same flesh type did not fall in the same subset, apart for the stony hard (tab. 5).

\section{CONCLUSIONS}

The possibility of applying the TRS technique to assess the texture phenotypes of peach fruit has been tested. The TRS technique allowed separating the effects of absorption and scattering properties. With the exception of the cultivar 'Iride', particularly rich in anthocyanins, and 'Ghiaccio', a totally de-pigmented fruit, the absorption spectra of all the samples were similar.

For what concerns scattering properties, by considering the two Mie parameters, $a$ and $b$, it was possible to discriminate three out of four texture types (melting, slow melting and stony hard), but not the non melting. Works are in progress in order to achieve further improvements that may lead to a full discrimination.

\section{Acknowledgements}

We wish to thank Stefano Foschi, CRPV Cesena (Italy) and Martina Lama (ASTRAInnovazione, Italy), for their assistance in field operations

\section{Literature Cited}

Cen, H., Lu, R., Mendoza, F.A., Ariana, D.P. Assessing multiple quality attributes of peaches using optical absorption and scattering properties. Transactions of the ASABE 55: 647-657

Cubeddu, R., Pifferi, A., Taroni, P., Torricelli, A.2002.Measuring fresh fruit and vegetable quality: advanced optical methods. In W Jongen, ed, Fruit and Vegetable Processing-Improving Quality. CRC Press/Woodhead Publishing Limited, Boca Raton, Boston, New York, Washington, DC/Cambridge (England), pp 150-169

Dahm, D.J., Dahm, K.D. 2001. The physics of near-infrared scattering. In AA of C Chemists., ed, Near-infrared Technology in the Agricultural and Food industries 2nd ed. P. Williams and K. Norris. St. Paul, Minn., pp 1-17

Lu, R., Peng, Y. 2006. Hyperspectral Scattering for assessing Peach Fruit Firmness. Biosystems Engineering 93: 161-171.

Mourant J. R., Fuselier T, Boyer J, Johnson T M \& Bigio I J (1997) Predictions and measurements of scattering and absorption over broad wavelength ranges in tissue phantoms Appl. Opt. 36 949-57.

Nilsson A M K, Sturesson C, Liu D L and Andersson-Engels S 1998 Changes in spectral shape of tissue optical properties in conjunction with laser-induced thermotherapy Appl. Opt. 37 1256-67 
Torricelli, A., Spinelli, L., Contini, D., Vanoli, M., Rizzolo, A., Eccher Zerbini, P. 2008.Time-resolved reflectance spectroscopy for non-destructive assessment of food quality. Sensing and Instrumentation for Food Quality and Safety 2: 82-89

\section{$\underline{\text { Tables }}$}

Table1. List of all the analyzed accessions

\begin{tabular}{l|l|l}
\hline \multicolumn{1}{c|}{ CULTIVAR } & HARVEST DATE & TEXTURE TYPE \\
\hline Alice Col & 13 July2011 & NM \\
BO000200006 & 28 July 2011 & NM \\
Iride & 8 July 2011 & NM \\
Dixired & 8 July 2011 & M \\
Glohaven & 22 July 2011 & M \\
Redhaven & 15 July 2011 & M \\
Ghiaccio & 21 July 2011 & SH \\
IFF 331 & 30 July 2011 & SH \\
Big Top & 15 July 2011 & SM \\
Rich Lady & 15 July 2011 & SM \\
Vistarich & 19 July 2011 & SM \\
\hline
\end{tabular}

Tab. 2. Scattering of the four phenotypes: $a$ parameter $\left(\mathrm{cm}^{-1}\right)$

\begin{tabular}{l|l|l|l|l}
\hline HSD di Tukey, & \multirow{2}{*}{$\mathrm{N}$} & \multicolumn{3}{|l}{ subset } \\
\cline { 3 - 5 } flesh type & & 1 & 2 & 3 \\
\hline melting & 90 & 16.27 & & \\
non melting & 90 & 16.35 & & \\
slow melting & 90 & & 17.60 & \\
stony hard & 60 & & & 18.56 \\
& & & & \\
\hline
\end{tabular}

Tab. 3. Scattering of the cultivars: $a$ parameter $\left(\mathrm{cm}^{-1}\right)$

\begin{tabular}{l|l|l|l|l|l|l}
\hline HSD di Tukey & \multirow{2}{*}{$\mathrm{N}$} & \multicolumn{3}{l}{ subset } \\
\cline { 3 - 7 } cv (flesh type) & & 1 & 2 & 3 & 4 & 5 \\
\hline Glohaven (M) & 30 & 13.04 & & & & \\
Iride (NM) & 30 & 13.72 & 13.72 & & & \\
Rich Lady (SM) & 30 & & 15.02 & & & \\
BO00020006 (NM) & 30 & & & 16.92 & & \\
Redhaven (M) & 30 & & & 17.07 & 17.07 & \\
Vistarich (SM) & 30 & & & 17.42 & 17.42 & \\
Alice Col (NM) & 30 & & & 18.39 & 18.39 & \\
IFF 331 (SH) & 30 & & & 18.45 & 18.45 & \\
Ghiaccio (SH) & 30 & & & 18.67 & 18.67 & 18.67 \\
Dixired (M) & 30 & & & & 18.72 & 18.72 \\
Big Top (SM) & 30 & & & & & 20.38 \\
& & & & & & \\
\hline
\end{tabular}


Tab. 4. Scattering of the four phenotypes: $b$ parameter.

\begin{tabular}{l|l|l|l|l}
\hline HSD di & N & \multicolumn{3}{|l}{ subset } \\
\cline { 3 - 5 } $\begin{array}{l}\text { Tukey } \\
\text { flesh type }\end{array}$ & & 1 & 2 & 3 \\
\hline melting & 90 & .27 & & \\
slow melting & 90 & & .36 & \\
non melting & 90 & & .37 & \\
stony hard & 60 & & & .54 \\
\hline
\end{tabular}

Tab. 5. Scattering of the cultivars: $b$ parameter .

\begin{tabular}{l|l|l|l|l|l|l|l}
\hline HSD di Tukey & \multirow{2}{*}{$\mathrm{N}$} & \multicolumn{2}{|l|}{ subset } \\
cv (flesh type) & & 1 & 2 & 3 & 4 & 5 & 6 \\
\hline Iride (NM) & 30 & .18 & & & & & \\
Glohaven (M) & 30 & .21 & .21 & & & & \\
Dixired (M) & 30 & & .28 & .28 & & & \\
Rich Lady (SM) & 30 & & .30 & .30 & .30 & & \\
Redhaven (M) & 30 & & & .31 & .31 & .31 & \\
Vistarich (SM) & 30 & & & & .37 & .37 & \\
Alice Col (NM) & 30 & & & & .38 & .38 & \\
Big Top (SM) & 30 & & & & & .40 & \\
Ghiaccio (SH) & 30 & & & & & & .53 \\
BO00020006 (NM) & 30 & & & & & & .53 \\
IFF331 (SH) & 30 & & & & & & .55 \\
& & & & & & & \\
\hline
\end{tabular}

\section{Figures}

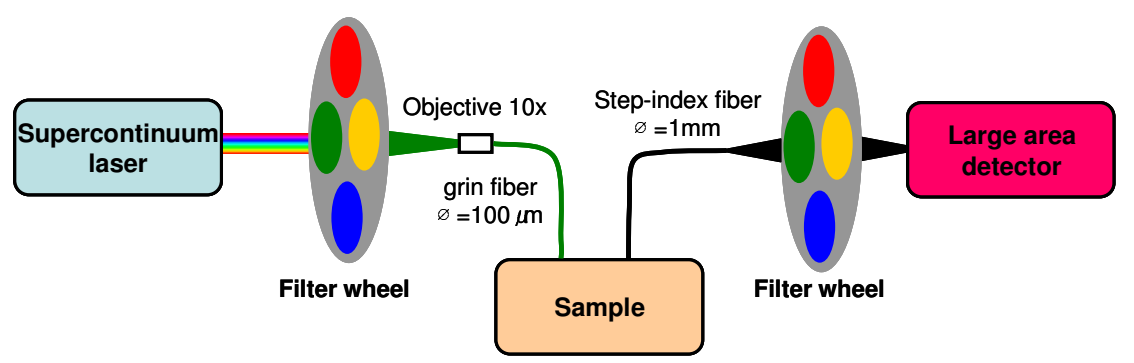

Fig. 1.Scheme of the TRS instrumental setup. 


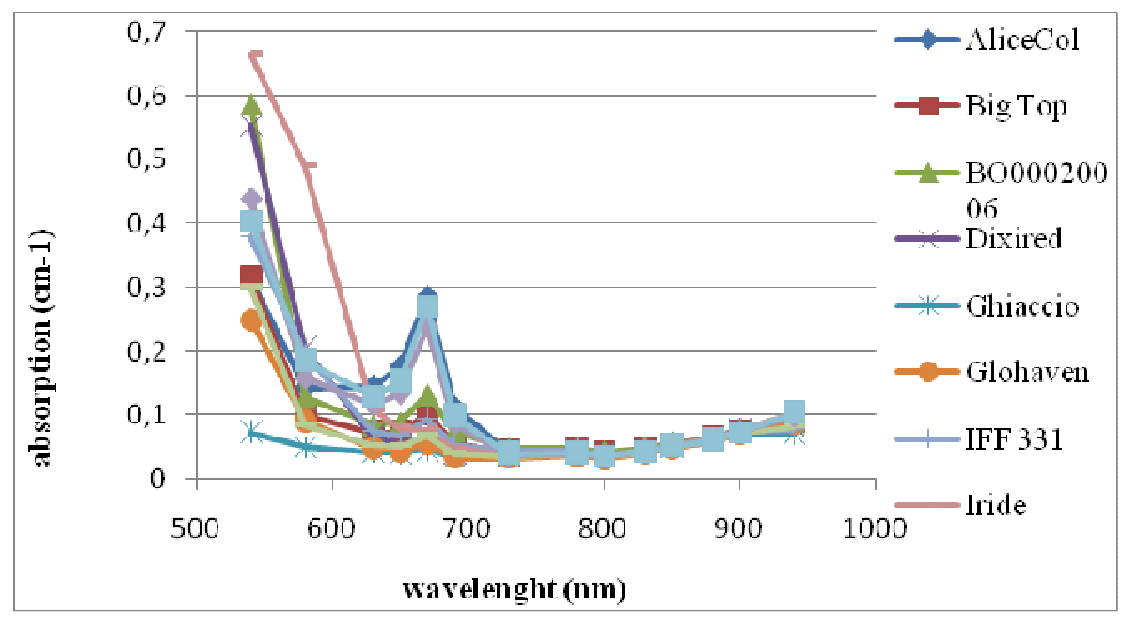

Fig. 2. Absorption spectra for different flesh phenotypes (average of 30 samples per each cultivar).

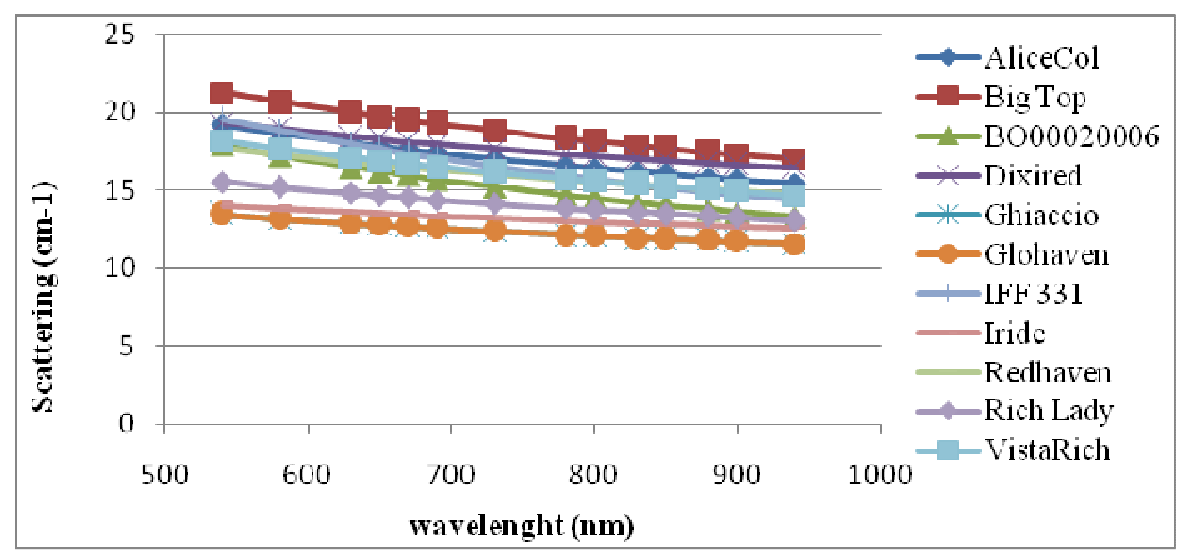

Fig. 3. Reduced scattering spectra for different flesh phenotypes (average of 30 samples per each cultivar).

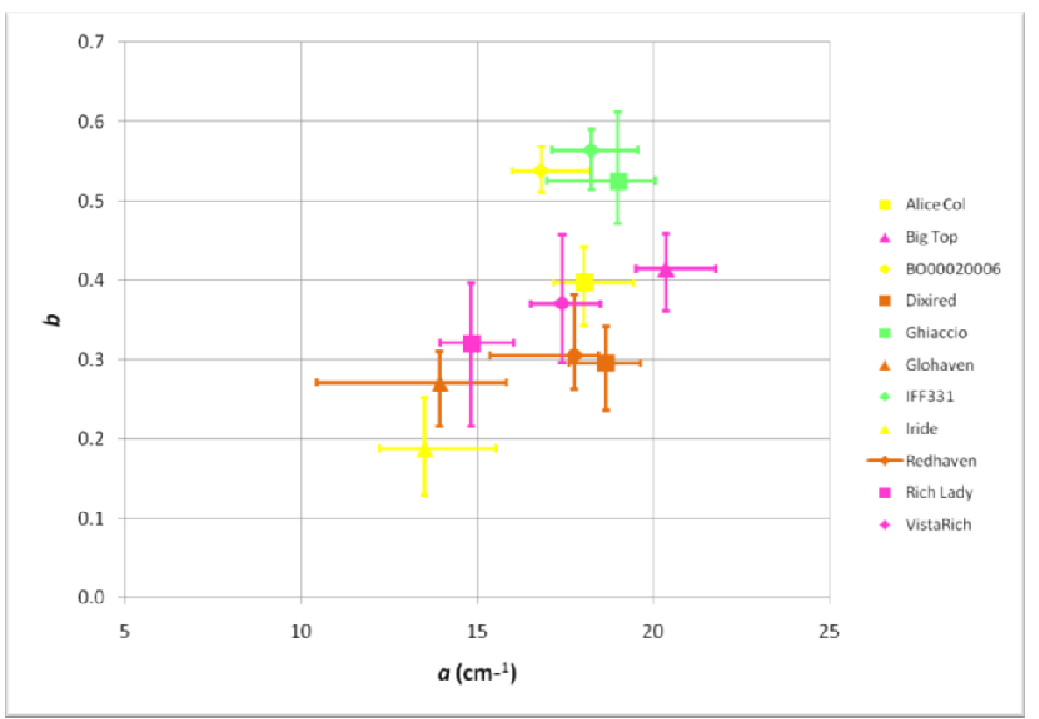

Fig. 4. Scatter plot of the equivalent Mie parameters for the different flesh phenotypes 